\title{
Topological line defects in graphene for applications in gas sensing
}

\author{
Fábio A.L. de Souza ${ }^{\mathrm{a}, \mathrm{b}}$, Rodrigo G. Amorim ${ }^{\mathrm{c}, ~ *}$, Jariyanee Prasongkit ${ }^{\mathrm{d}, \mathrm{e}}$, \\ Wanderlã L. Scopel ${ }^{\mathrm{b}}$, Ralph H. Scheicher ${ }^{\mathrm{f}}$, Alexandre R. Rocha ${ }^{\mathrm{g}, \mathrm{h}}$ \\ ${ }^{a}$ Federal Institute of Education, Science and Technology of Espírito Santo, Ibatiba, ES, Brazil \\ b Departamento de Física, Universidade Federal do Espírito Santo, Vitória, ES, Brazil \\ ${ }^{c}$ Departamento de Física, ICEx, Universidade Federal Fluminense, Volta Redonda, RJ, Brazil \\ d Division of Physics, Faculty of Science, Nakhon Phanom University, Nakhon Phanom, 48000, Thailand \\ e Nanotec-KKU Center of Excellence on Advanced Nanomaterials for Energy Production and Storage, Khon Kaen 40002, Thailand \\ ${ }^{\mathrm{f}}$ Division of Materials Theory, Department of Physics and Astronomy, Uppsala University, SE-751 20 Uppsala, Sweden \\ ${ }^{\mathrm{g}}$ Instituto de Física Teórica, Universidade Estadual Paulista, São Paulo, Brazil \\ ${ }^{\mathrm{h}}$ Department of Chemical Engineering, Massachusetts Institute of Technology, Cambridge, MA 02139, USA
}

\section{A R T I C L E I N F O}

\section{Article history:}

Received 7 June 2017

Received in revised form

9 November 2017

Accepted 12 November 2017

Available online 25 December 2017

\section{Keywords:}

Nanosensor

Graphene

Electronic transport

\begin{abstract}
A B S T R A C T
Topological line defects in graphene synthesized in a highly controlled manner open up new research directions for nanodevice applications. Here, we investigate two types of extended line defects in graphene, namely octagonal/pentagonal and heptagonal/pentagonal reconstructions. A combination of density functional theory and non-equilibrium Green's function methods was utilized in order to explore the application potential of this system as an electronic gas sensor. Our findings show that the electric current is confined to the line defect through gate voltage control, which combined with the enhanced chemical reactivity at the grain boundary, makes this system a highly promising candidate for gas sensor applications. As a proof of principle, we evaluated the sensitivity of a prototypical device toward $\mathrm{NO}_{2}$ molecule, demonstrating that it is indeed possible to reliably detect the target molecule.
\end{abstract}

() 2017 Elsevier Ltd. All rights reserved.

\section{Introduction}

The most widely used method for production of large scale graphene sheets is chemical vapor deposition (CVD) on metal surfaces [1]. Previous works have observed grains, grain boundaries (GB) and topological defects in CVD-grown graphene using transmission electron microscopy [2-4]. Due to the honeycomb structure of graphene, the GB's are usually composed of octagonal, pentagonal, heptagonal carbon rings and Stone-Wales (SW) reconstructions [2,5].

In 2010, Lahiri et al. synthesized, on a $\mathrm{Ni}(111)$ substrate, a graphene sheet enclosing an extended one-dimensional defect containing octagonal and paired pentagonal $s p^{2}$-hybridized carbon rings [3]. Subsequently, it was shown that these octagonalpentagon (o-p) defects can be produced in free-standing graphene in a controlled way, through simultaneous electron irradiation and Joule heating by an applied electric current [6].

\footnotetext{
* Corresponding author.

E-mail addresses: fabio.souza@ifes.edu.br (F.A.L. de Souza),rgamorim@id.uff.br (R.G. Amorim)
}

Remarkably, such extended line defects induce a charge redistribution in their surroundings, and could thus behave as a metallic wire embedded in a graphene sheet [3]. Therefore, in contrast to isolated defects, which are in general associated with electron scattering and heat generation [7], such topological defects could actually enhance the electronic transport properties of graphene [5,8-10], although it should be mentioned that these properties are generally highly dependent on the relative orientation between grain boundary and transport direction [11-13].

In 2007, Schedin et al. [14] have demonstrated the capability of detecting a single molecule adsorbed on a graphene-based sensor at room temperature. It was shown that the interaction between graphene and electron acceptor molecules (e.g., $\mathrm{H}_{2} \mathrm{O}$ and $\mathrm{NO}_{2}$ ) increases the device conductivity, whereas electron donors (e.g., $\mathrm{CO}$ and $\mathrm{NH}_{2}$ ) induce a decrease in conductivity, an effect that has also been experimentally observed for a variety of other molecules [15-19]. Nonetheless, interactions between graphene and closed shell molecules tend to be relatively weak, which implies a low signal to noise ratio. Zhang et al., on the other hand, have shown, via $a b$ initio calculations, that the sensitivity of graphene-based nanosensors could be improved by introducing dopants or 
vacancies [20,21]. However, such an improvement to graphenebased gas sensors comes at the cost of significantly larger adsorption energies of the target molecules, and consequently to an increase in recovery time [20].

Thus, a good sensor should have three basic features: i) a small but not negligible binding energy $E_{b}$, since recovery time is correlated to $E_{b}$. In other words, the larger the binding energy, the harder it is to remove it after detection, rendering the device nonresponsive after a single use. Furthermore, the sensor should present significant ii) sensitivity, and iii) selectivity to different gases [22-24]. In our case, this means large changes in conductance upon adsorption for a particular gas compared to others. Extended line defects in graphene possess higher chemical reactivity compared to pristine regions $[25,26]$, but significantly lower than single point defects. This energy window can allow for an enhanced response for chemical or sensing applications [27], and therefore pose as interesting alternatives to modified graphene-based gas sensors. In this work, we have used a combination of density functional theory and non-equilibrium [28] Green's functions [29,30] to investigate the electronic and transport properties of the two different types of line defects in graphene. While the octagonal-pentagonal (o-p) defect has been experimentally synthesized, it is possible that other topological line defects can form as well in graphene. One such proposal was presented by Berger [31] based on theoretical predictions. This defect consists of heptagonal and pentagonal rings (h-p). We demonstrate that these extended defects can behave as one-dimensional wires embedded in a pristine graphene sheet and that, by applying an appropriate gate voltage, it is possible to select which part of the device the electric current will predominantly flow through. This, combined with the enhanced chemical reactivity of atomic sites along the line defects, yields high promise for this system to act as a highly sensitive and selective gas sensor. The feasibility of such topological defects to detect and identify gas molecules is explored here for $\mathrm{NO}_{2}$ as a point in case. We initially examined the energetic stability of $\mathrm{NO}_{2}$ on both o-p and h-p line defects and found that the target molecule binds more strongly to the former system, by approximately $0.6 \mathrm{eV}$ per molecule. Additionally, analysis of the conductance changes induced by the adsorbate on the prototypical device has shown that o-p exhibits higher sensitivity towards $\mathrm{NO}_{2}$ than h-p.

\section{Methodology}

The structures of the topological defects considered in this work are shown in Fig. 1(a-b). All calculations were performed using periodic boundary conditions in the $x$-direction. We ensure that there is no interaction between images of the line defects. In that way the o-p (h-p) supercell is 18.46 (19.47) $\AA$ wide, and 29.52 (29.28) $\AA$ long. In both systems, the distance between the line defect and its in-plane periodic image is greater than $14 \AA$, which is sufficient to avoid spurious interactions. Geometry relaxations and electronic structure calculations were performed using density functional theory (DFT) [32,33] in the generalized gradient approximation of Perdew, Burke, and Ernzerhof (GGA-PBE) [34], which was corrected to take into account van der Waals interactions [35,36], as implemented in the SIESTA code [37]. A double-zeta basis set with polarization orbitals (DZP) along with norm-conserving pseudopotentials [38] was employed for all the atoms. The mesh cutoff was set to 200Ry for the real space grid, and the Monkhorst-Pack scheme [39] was used with a mesh of $4 \times 1 \times$ $3 k$-points for the Brillouin zone integration. Structural relaxations were carried out until residual forces on each ion were less than $0.01 \mathrm{eV} / \AA$.

The electronic transport properties were investigated via the non-equilibrium Green's function (NEGF) method [29,30,40,41]. a)
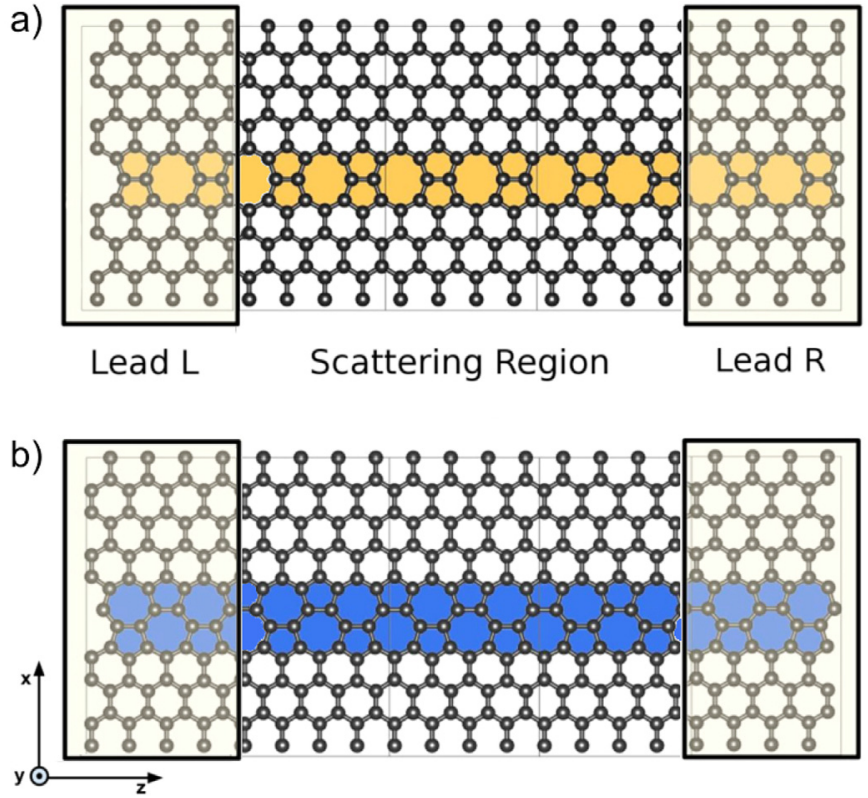

Fig. 1. A sketch of the model used to calculate electronic transport properties of o-p device (upper panel) and h-p device (lower panel). Carbon atoms within the shaded regions forming the connecting wires of the left and right lead are referred to as $C_{w}$ while the remaining carbon atoms associated with pristine graphene are labelled $C_{g}$. $(A$ colour version of this figure can be viewed online.)

Within that approach, the system is divided into three parts: a scattering region (SR) enclosed by two semi-infinite electrodes on either side [42]. The problem can then be treated as a scattering problem [43]. The main quantity that can be used to describe the system is the retarded Green's function

$G^{R}(E, V)=\lim _{\eta \rightarrow 0^{+}}\left[\varepsilon S_{\mathrm{SR}}-H_{\mathrm{SR}}-\Sigma_{\mathrm{L}}(E, V)-\Sigma_{\mathrm{R}}(E, V)\right]^{-1}$,

where $\varepsilon=E+i \eta, S_{\mathrm{SR}}$ and $H_{\mathrm{SR}}$ are the overlap and Hamiltonian for the scattering region, and $\Sigma(E, V)_{\mathrm{L} / \mathrm{R}}$ are the so-called self-energies, which include the effects of the electrodes onto the SR. In this work the Hamiltonian for the scattering region is taken as the KohnSham Hamiltonian, which is a functional of the charge density $n(\mathbf{r}), H_{\mathrm{SR}} \equiv H_{\mathrm{KS}}[n]$.

For a given bias $V$, the two electrodes have fixed chemical potentials $\mu_{\mathrm{L} / \mathrm{R}}=\mu_{0 \pm V} / 2$, which define the boundary conditions for the problem. Thus, a self-consistent calculation is performed to find the charge density at steady state, and consequently the Hamiltonian. From that, the current can be calculated

$I(V)=\frac{2 e}{h} \int T(E, V)\left(f\left(E-\mu_{L}\right)-f\left(E-\mu_{R}\right)\right) d E$

where the quantity

$T(E)=\operatorname{Tr}\left[\Gamma_{\mathrm{R}}(E, V) G^{\mathrm{R}}(E, V) \Gamma_{\mathrm{L}}(E, V) G^{\mathrm{A}}(E, V)\right]$,

is the probability that an incoming electron with energy $E$, at a given bias $V$ will be transmitted across the scattering region, and $f(\varepsilon)$ is the Fermi-Dirac distribution for energy $\varepsilon$. The operators $\Gamma_{L,(R)}(E, V)=i\left[\Sigma_{L,(R)}-\Sigma_{L,(R)}^{\dagger}\right]$ define the coupling with the left and right electrodes.

In the limit of zero bias (linear regime), the conductance can be related to the transmission $T(E, 0)$ via the Fisher-Lee relation [44]. 


$$
G=G_{0} T\left(E_{F}\right),
$$

where $G_{0}=2 e^{2} / h$ is the quantum of conductance. At biases different from zero, the current corresponds to the integral over all transmission probabilities within the energy window around the Fermi energy defined by $\pm V / 2$ [41]. Finally, by projecting the transmission between each pair of adjacent sites, one can gain insight into the pathway of the local current flow through the device; hence, the local current density between sites $N$ and $M$ of the scattering region is given by:

$$
i(E)_{N \rightarrow M}=4 \frac{e}{h} \sum_{\substack{n \in N \\ m \in M}} \Im\left[\left\{G^{R}(E) \Gamma_{\mathrm{L}} G^{A}(E)\right\}_{m n} H_{n m}\right],
$$

where the above sum runs over all localized atomic orbitals $n$ and $m$ of the basis set belonging to atoms in either site, and for a specific energy $E$. For further details, we refer to the works of Okabayashi et al. [45] and of Paulsson and Brandbyge [46].

\section{Results and discussion}

Focusing on the electronic and transport properties of the two different line defects, Fig. 2a shows the zero-bias transmission spectra for both systems studied herein. In the case of the o-p line defect (solid line), one can observe that for an energy window of approximately $0.6 \mathrm{eV}$ around the Fermi level $\left(E_{F}\right)$ there are two conducting channels which are ascribed to states associated with carbon atoms from the line defect $\left(C_{w}\right)$ as well as from the pristine graphene region $\left(C_{g}\right)$, as can be seen in the projected density of states (PDOS) of Fig. 2b. We also note that in the two energy ranges from -1.00 to $-0.20 \mathrm{eV}$ and from 0.10 to $0.20 \mathrm{eV}$, one extra conducting path appears leading to 3 channels. Additionally, a 1channel plateau between 0.20 and $0.90 \mathrm{eV}$ appears, which can be projected onto states from both $C_{g}$ and $C_{w}$ atoms.

Considering the h-p defect device (dashed line in Fig. 2a), zerotransmission is observed within the range $E_{F} \pm 0.20 \mathrm{eV}$, which is in line with the band-gap observed in the PDOS (Fig. 2c). For higher energies, however, h-p presents a transmission enhancement mimicking its PDOS for those corresponding energy ranges (see Fig. 2c). It is worth noting, particularly in the case of the h-p defect, that in the limit of an infinitely wide graphene sheet one should expect no gap. The key point is that, as the graphene sheet widens, the gap will monotonically close and the relative density of states between atoms in the wires and in the sheets will remain unchanged (see Supplementary data for further details).

To further understand the zero-bias transmission curves in Fig. 2a, and shed light on the way electrons flow through the devices, local currents are analyzed at specific energy values. In Fig. 3 the local currents at $E=-0.3,0.0$ and $+0.3 \mathrm{eV}$, (upper panels correspond to the o-p and lower panels to the h-p system), are shown. At the Fermi level (Fig. 3-b), the local currents spread somewhat uniformly over the o-p system, since the contribution for the transmission is shared by states from $C_{g}$ and $C_{w}$ atoms (as seen in PDOS of Fig. 2b). On the other hand, for the h-p device there is no current due to the energy gap of approximately $0.9 \mathrm{eV}$, and to the fact that the density of states in graphene goes to zero exactly at $E_{F}$.

The more interesting behavior occurs away from the Fermi level, in regions which could be assessed by a gate voltage. This could be achieved by depositing graphene containing the line defects onto a substrate, which would not alter the properties of the system [47]. A back-gate can then be used to alter the position of its Fermi level [47]. In the o-p case at $\mathrm{E}= \pm 0.3 \mathrm{eV}$ the current is confined to the wire. It is important to note that this does not mean that the current in graphene is zero, but indicates that the current density is significantly larger than in the wire. In fact, the local currents in the graphene are barely visible. We note that in the o-p case with $\mathrm{E}=0.3 \mathrm{eV}$ the PDOS indicates that the density of states for both $C_{g}$ and $C_{w}$ atoms is similar. The total current is, in fact, similar, but for the line defect it is confined in a much narrower region giving rise to a higher current density. A similar effect is also observed in the hp case for $\mathrm{E}=+0.3 \mathrm{eV}$, albeit here, the PDOS for $C_{w}$ is significantly higher. The opposite trend is present for $\mathrm{E}=-0.3 \mathrm{eV}$, i.e. the wire is insulating whereas the graphene matrix is conducting for negative energies. These findings corroborate the idea that the o-p extended defect can act as a metallic wire embedded in graphene [3,27,48].

Considering the results above, we then turn to the use of these devices for sensing. As previously mentioned, a key problem in using graphene for sensing applications is the fact that the binding energy of closed-shell molecules on graphene is usually small. Although this leads to easy recovery, it usually decreases the signal

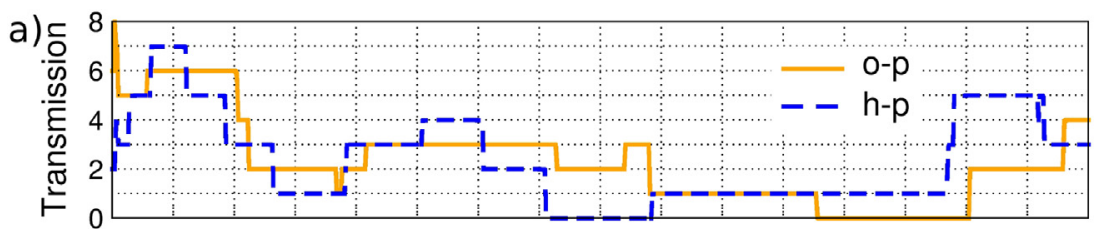

b)

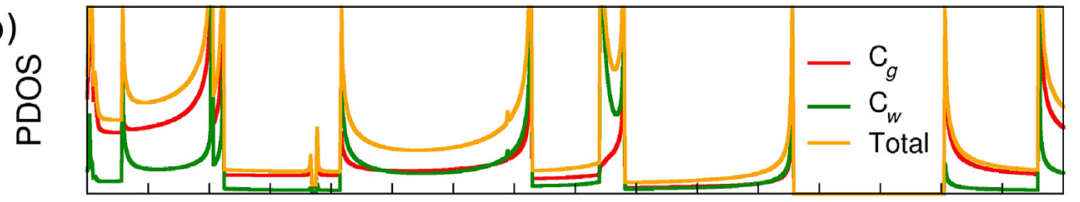

C)

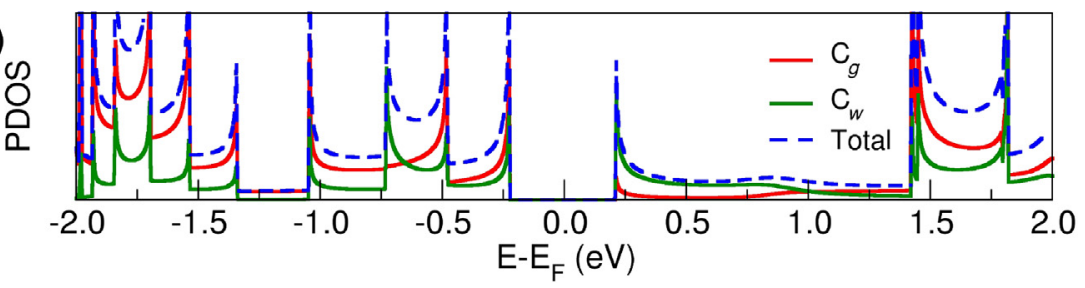

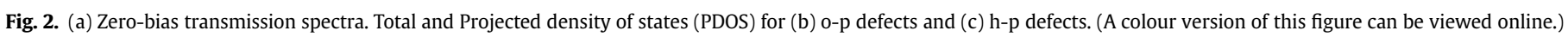




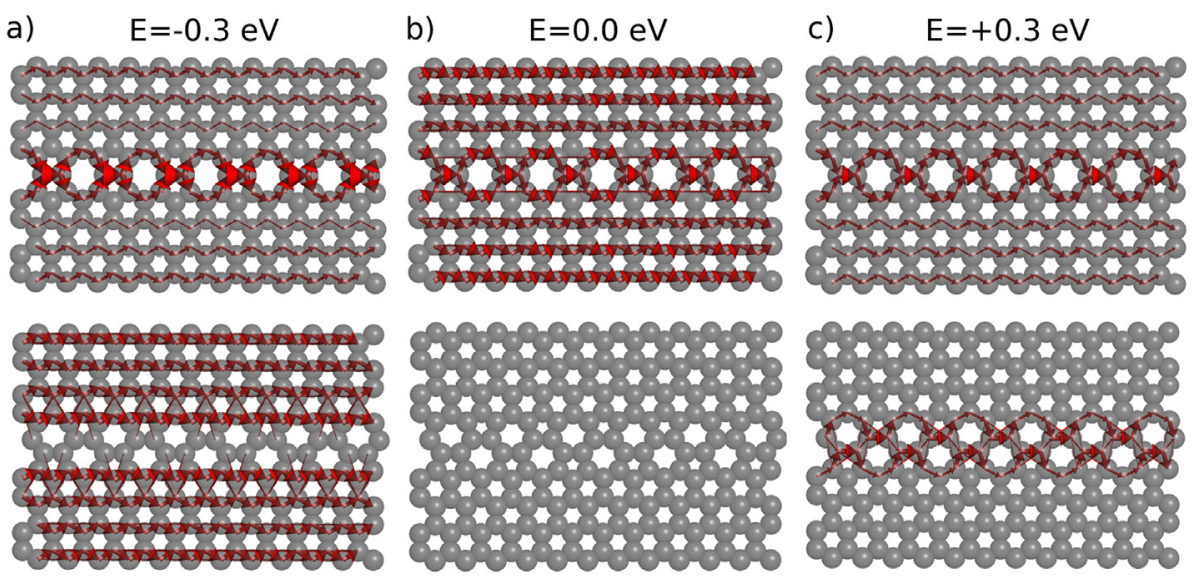

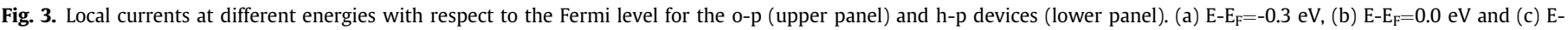
$\mathrm{E}_{\mathrm{F}}=+0.3 \mathrm{eV}$. (A colour version of this figure can be viewed online.)

to noise ratio. Furthermore, in a two-dimensional system, there is normally a large number of conducting channels available. In that case, to achieve large sensitivity, scatterers must couple to a large number of k-vectors for any given energy. On the other hand, the capability of controlling which device region will be associated with current flow (essentially creating a one-dimensional wire), combined with the enhanced reactivity of sites in extended topological defects in graphene $[25,26]$, could make o-p and h-p systems promising candidates for bio- and gas sensor applications. Although both systems studied here are stable from the theoretical point of view [31,49,50], the o-p line defect has been synthesized in a highly controlled manner [3,6] and has a lower formation energy.

Focusing on the latter defect, and in order to get a deeper insight into the feasibility of gas sensors based on graphene grain boundaries, the energetic stability, charge transfer and sensitivity of o-p system toward $\mathrm{NO}_{2}$ adsorption was evaluated. We performed calculations for the adsorption of $\mathrm{NO}_{2}$ molecule on different positions of the line defects (see supplementary data for details). Fig. 4a (left panel) shows the fully relaxed geometry for $\mathrm{NO}_{2}$ on the o-p device. It is worth noting that the $\mathrm{NO}_{2}$ is located on top of a carbon site shared by the paired pentagon and the octagon carbon ring, which is approximately $0.13 \mathrm{eV}$ more favorable than the competing ones (see supplemental data). The binding energy of the $\mathrm{NO}_{2}$ molecule on o-p device was $0.61 \mathrm{eV} /$ molecule, which is approximately $0.13 \mathrm{eV} /$ molecule more stable than pristine graphene. In contrast, it is $2.42 \mathrm{eV} /$ molecule smaller than defective graphene [20] where a covalent bond was found.

As a first step to understand the interaction between molecule and the line defect - and its subsequent effect on transport - we have mapped the total charge density redistribution $\Delta \rho(\vec{r})$ by comparing the total charge of the system $\rho_{\left(\mathrm{NO}_{2}+\text { device }\right)}(\vec{r})$ with its separated parts, $\rho_{\mathrm{NO}_{2}}(\vec{r})$ and $\rho_{\text {device }}(\vec{r})$,

$$
\Delta \rho(\vec{r})=\rho_{\text {device }+\mathrm{NO}_{2}}(\vec{r})-\left(\rho_{\mathrm{NO}_{2}}(\vec{r})+\rho_{\text {device }}(\vec{r})\right)
$$

Our results for $\Delta \rho(\vec{r})$ are depicted in Fig. 4a (right panel). A uniform charge density redistribution is found on the plane of the $C$ atoms around the $\mathrm{C}-\mathrm{N}$ bond with gain (yellow) and loss (green) of electronic charge. We also note a significant charge density redistribution in the region of the $\mathrm{C}-\mathrm{N}$ bond perpendicular to the plane of $C$ atoms. Based on the Bader scheme [51,52] we found that the net charge of the o-p system was reduced by $0.23 e$, which indicates that the $\mathrm{NO}_{2}$ molecule is an electron acceptor, as already predicted for $\mathrm{NO}_{2}$ on defective and pristine graphene [20].

Both the relatively small $E_{b}$ and large charge transfer are good
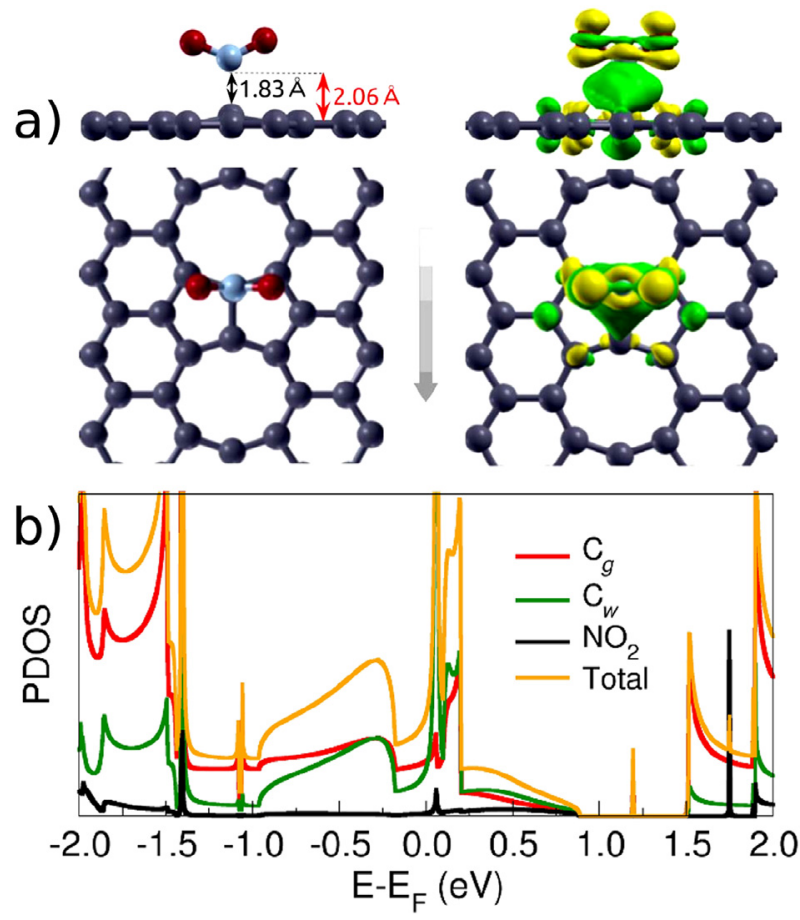

Fig. 4. (a) Fully relaxed structure of $\mathrm{o}-\mathrm{p}+\mathrm{NO}_{2}$ (left panel) and electronic charge density redistribution (right panel), colored according to sign, yellow for positive and green for negative (isosurface for a value of $0.003 \mathrm{e} \times \mathrm{Bohr}^{-3}$ ), which is calculated as the difference between the charge density of the total system $\left(0-\mathrm{p}+\mathrm{NO}_{2}\right)$ and that of each constituent part $\left(0-p, \mathrm{NO}_{2}\right.$ ) in separate. (b) o-p $+\mathrm{NO}_{2}$ PDOS. (A colour version of this figure can be viewed online.)

indicators that this type of device would satisfy our two prerequisites. In particular, the large charge transfer is an indication that the interaction between molecule and device is large enough to yield noticeable changes in the transport properties.

Next, we explore the electronic transport and define the zerobias sensitivity as

$S(\%)=\left|\frac{\left(g_{0}-g\right)}{g_{0}}\right| \times 100$

where $g_{0}$ stands for the conductance of the o-p system (used as a reference) and $g$ for the system with the adsorbed molecule (o-p 

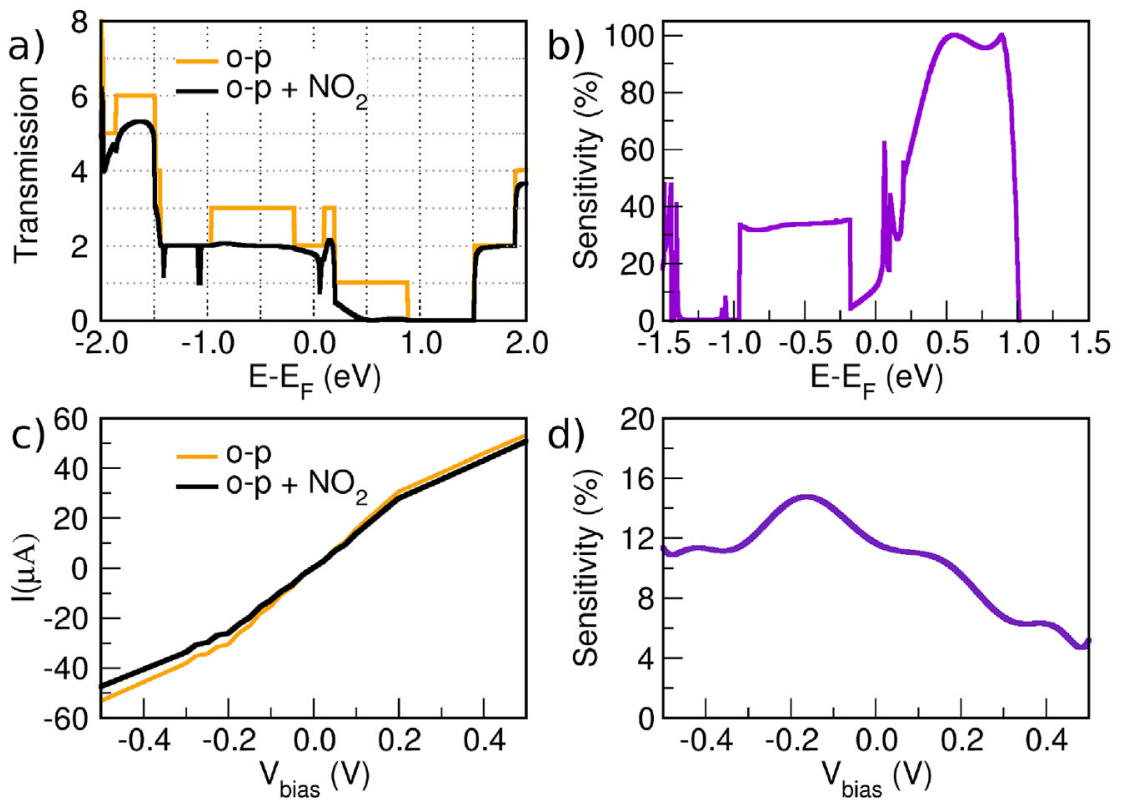

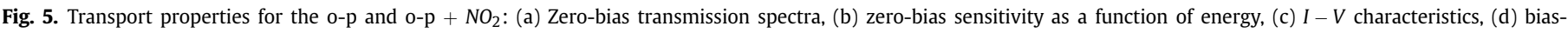
dependent sensitivity. (A colour version of this figure can be viewed online.)

$+\mathrm{NO}_{2}$ ). The zero-bias transmission for both cases is depicted in Fig. 5a. We can note a significant drop in transmission upon adsorption. At least three anti-resonance peaks are present; two of those (at -1.4 and $0.1 \mathrm{eV}$ ) are due to the molecule and one $(-1.1 \mathrm{eV})$ is associated with interaction-induced localized states on the device as shown in the PDOS of Fig. 4-b, i.e. the states from the molecule are hybridized with the defective carbon atoms.

The zero-bias sensitivity as a function of energy is depicted in Fig. 5-b. This is equivalent to tuning the Fermi energy by a gate voltage. We observed a significant - and approximately constant sensitivity ( $\sim 35 \%)$ for a gate voltage in the range of $(-1.00$ to -0.25 $\mathrm{eV})$, and very large sensitivities for $(\sim 60-100 \%)$ from 0.25 to $1.00 \mathrm{eV}$. In both cases we note that the interaction with the molecule leads to the complete suppression of one conductance channel. For the resonance close to the Fermi energy, the sensitivity is approximately $60 \%$. As pointed out before, this resonance comes from the gas molecule states hybridized with the defect's $C$ atoms. Fig. 5c shows the $I-V$ characteristics for our setups. We note that the $I-V$ characteristics upon adsorption are not symmetric with respect to bias. While the isolated molecule and the o-p defect are mirror-symmetric, upon adsorption, that symmetry is broken. Different self-consistent responses to an external bias lead to a small asymmetry due to charging. Fig. $5 \mathrm{~d}$ shows the behavior of the bias-dependent sensitivity, which is calculated using equation (7) by replacing the conductance by the corresponding currents at each bias voltage. which, for negative applied voltages, shows a larger difference compared to positive ones. Although the calculated sensitivity is small $-\sim 8-10 \%$ - it is similar to the zero-bias transmission in the energy window $\pm 0.25 \mathrm{eV}$. This means that at low bias, it compares well with the zero-bias transmission. We thus note that it could be significantly enhanced by changing the gate voltage accessing regions where the defect is more metallic in nature. Finally, regarding selectivity, we note that the binding energy of $\mathrm{NO}_{2}$ [20] is higher than that of $\mathrm{O}_{2}$ [53]; this not only influences the residence time, but also the electronic transport [54], thus possibly leading to good selectivity as well.

\section{Conclusions}

In conclusion, electronic and transport properties of extended line defects in graphene have been investigated. We demonstrated that such topological defects can work as a metallic wire embedded in perfect graphene, and by applying specific gate voltages one is able to control through which part of the modified graphene sheet the electric current will most likely flow. The ability of controlling current pathways along with the enhanced reactivity of sites at defective regions make these systems promising candidates to be used in advanced gas sensor platforms. Based on our results, it was shown that a device based on the octagonal-pentagonal (o-p) defect is highly sensitive towards $\mathrm{NO}_{2}$ molecule and constitutes an interesting alternative to improve graphene gas sensor capabilities without detrimentally affecting its recovery time.

\section{Acknowledgement}

The authors acknowledge support from Brazilian agencies FAPES, FAPESP (Project \# 2016/01343-7 and 2015/26862-4), and CNPq. ARR acknowledges support from the ICTP-Simmons Foundation associate scheme. JP thanks Thailand Research Fund (MRG5980185) and the National Nanotechnology Center (NANOTEC), NSTDA, Ministry of Science and Technology, Thailand, through its program of Centers of Excellence Network. Financial support from the Carl Tryggers Foundation (for RGA.) and the Swedish Research Council (for RHS) is also gratefully acknowledged. The Swedish National Infrastructure for Computing (SNIC), CENAPAD-SP and the Uppsala Multidisciplinary Center for Advanced Computational Science (UPPMAX) provided computing time for this project.

\section{Appendix A. Supplementary data}

Supplementary data related to this article can be found at https://doi.org/10.1016/j.carbon.2017.11.029. 


\section{References}

[1] S. Bae, H. Kim, Y. Lee, X. Xu, J.-S. Park, Y. Zheng, J. Balakrishnan, T. Lei, H.R. Kim, Y.I. Song, Y.-J. Kim, K.S. Kim, B. Özylmaz, J.-H. Ahn, B.H. Hong, S. Iijima, Roll-toroll production of 30-inch graphene films for transparent electrodes, Nat. Nanotechnol. 5 (2010) 574-578.

[2] P.Y. Huang, C.S. Ruiz-Vargas, A.M. van der Zande, W.S. Whitney, M.P. Levendorf, J.W. Kevek, S. Garg, J.S. Alden, C.J. Hustedt, Y. Zhu, J. Park, P.L. McEuen, D.A. Muller, Grains and grain boundaries in single-layer graphene atomic patchwork quilts, Nature 469 (2011) 389-392.

[3] J. Lahiri, Y. Lin, P. Bozkurt, I.I. Oleynik, M. Batzill, An extended defect in graphene as a metallic wire, Nat. Nanotechnol. 5 (2010) 326-329.

[4] K. Kim, Z. Lee, W. Regan, C. Kisielowski, M. Crommie, A. Zettl, Grain boundary mapping in polycrystalline graphene, ACS Nano 5 (2011) 2142-2146.

[5] L. Vicarelli, S.J. Heerema, C. Dekker, H.W. Zandbergen, Controlling defects in graphene for optimizing the electrical properties of graphene nanodevices, ACS Nano 9 (2015) 3428-3435.

[6] J.-H. Chen, G. Autès, N. Alem, F. Gargiulo, A. Gautam, M. Linck, C. Kisielowski, O. Yazyev, S. Louie, A. Zettl, Controlled growth of a line defect in graphene and implications for gate-tunable valley filtering, Phys. Rev. B 89 (2014), 121407.

[7] S. Haldar, R.G. Amorim, B. Sanyal, R.H. Scheicher, A.R. Rocha, Energetic stability, stm fingerprints and electronic transport properties of defects in graphene and silicene, RSC Adv. 6 (2016) 6702-6708.

[8] O.V. Yazyev, Y.P. Chen, Polycrystalline graphene and other two-dimensional materials, Nat. Nanotechnol. 9 (2014) 755-767.

[9] O.V. Yazyev, S.G. Louie, Topological defects in graphene: dislocations and grain boundaries, Phys. Rev. B 81 (2010a), 195420.

[10] O.V. Yazyev, S.G. Louie, Electronic transport in polycrystalline graphene, Nat. Mater. 9 (2010b) 806-809.

[11] A.W. Tsen, L. Brown, M.P. Levendorf, F. Ghahari, P.Y. Huang, R.W. Havener, C.S. Ruiz-Vargas, D.A. Muller, P. Kim, J. Park, Tailoring electrical transport across grain boundaries in polycrystalline graphene, Science 336 (2012) 1143-1146.

[12] C. Páez, A. Pereira, J. Rodrigues, N. Peres, Electronic transport across linear defects in graphene, Phys. Rev. B 92 (2015), 045426.

[13] J. Sun, N. Lin, Z. Li, H. Ren, C. Tang, X. Zhao, Electronic and transport properties of graphene with grain boundaries, RSC Adv. 6 (2016) 1090-1097.

[14] F. Schedin, A. Geim, S. Morozov, E. Hill, P. Blake, M. Katsnelson, K. Novoselov, Detection of individual gas molecules adsorbed on graphene, Nat. Mater. 6 (2007) 652-655.

[15] H.E. Romero, P. Joshi, A.K. Gupta, H.R. Gutierrez, M.W. Cole, S.A. Tadigadapa, P.C. Eklund, Adsorption of ammonia on graphene, Nanotechnology 20 (2009) 245501.

[16] G. Ko, H.-Y. Kim, J. Ahn, Y.-M. Park, K.-Y. Lee, J. Kim, Graphene-based nitrogen dioxide gas sensors, Curr. Appl. Phys. 10 (2010) 1002-1004.

[17] C. Chen, S. Hung, M. Yang, C. Yeh, C. Wu, G. Chi, F. Ren, S. Pearton, Oxygen sensors made by monolayer graphene under room temperature, Appl. Phys. Lett. 99 (2011), 243502.

[18] H.J. Yoon, J.H. Yang, Z. Zhou, S.S. Yang, M.M.-C. Cheng, Carbon dioxide gas sensor using a graphene sheets, Sensor. Actuator. B Chem. 157 (2011) $310-313$.

[19] G.T. Feliciano, C. Sanz-Navarro, M.D. Coutinho-Neto, P. Ordejón, R.H. Scheicher, A.R. Rocha, Capacitive DNA detection driven by electronic charge fluctuations in a graphene nanopore, Phys. Rev. Appl. 3 (2015), 034003.

[20] Y.-H. Zhang, Y.-B. Chen, K.-G. Zhou, C.-H. Liu, J. Zeng, H.-L. Zhang, Y. Peng, Improving gas sensing properties of graphene by introducing dopants and defects: a first-principles study, Nanotechnology 20 (2009), 185504.

[21] Y.-H. Zhang, L.-F. Han, Y.-H. Xiao, D.-Z. Jia, Z.-H. Guo, F. Li, Understanding dopant and defect effect on $\mathrm{H}_{2} \mathrm{~S}$ sensing performances of graphene: a firstprinciples study, Comput. Mater. Sci. 69 (2013) 222-228.

[22] A.R. Rocha, M. Rossi, A. Fazzio, A.J.R. da Silva, Designing real nanotube-based gas sensors, Phys. Rev. Lett. 100 (2008), 176803.

[23] A.R. Rocha, M. Rossi, A.J.R. da Silva, A. Fazzio, Realistic calculations of carbonbased disordered systems, J. Phys. Appl. Phys. 43 (2010), 374002.

[24] R.G. Amorim, A. Fazzio, A.J.R. da Silva, A.R. Rocha, Confinement effects and why carbon nanotube bundles can work as gas sensors, Nanoscale 5 (2013) $2798-2803$

[25] K. Kim, R.W. Johnson, J.T. Tanskanen, N. Liu, M.-G. Kim, C. Pang, C. Ahn, S.F. Bent, Z. Bao, Selective metal deposition at graphene line defects by atomic layer deposition, Nat. Commun. 5 (2014).
[26] W. Brito, R. Kagimura, R. Miwa, Hydrogenated grain boundaries in graphene Appl. Phys. Lett. 98 (2011), 213107.

[27] A.R. Botello-Mendez, X. Declerck, M. Terrones, H. Terrones, J.-C. Charlier, Onedimensional extended lines of divacancy defects in graphene, Nanoscale 3 (2011) 2868-2872.

[28] M. Di Ventra, Electrical Transport in Nanoscale Systems, 1 edition, Cambridge University Press, 2008.

[29] M. Brandbyge, J. Mozos, P. Ordejón, J. Taylor, K. Stokbro, Density-functional method for nonequilibrium electron transport, Phys. Rev. B 65 (2002), 165401.

[30] A. Rocha, V. García-Suárez, S. Bailey, C. Lambert, J. Ferrer, S. Sanvito, Spin and molecular electronics in atomically generated orbital landscapes, Phys. Rev. B 73 (2006), 085414.

[31] D. Berger, C. Ratsch, Line defects in graphene: how doping affects the electronic and mechanical properties, Phys. Rev. B 93 (2016), 235441.

[32] P. Hohenberg, W. Kohn, Inhomogeneous electron gas, Phys. Rev. 136 (1964) B864.

[33] W. Kohn, L.J. Sham, Self-consistent equations including exchange and correlation effects, Phys. Rev. 140 (1965), A1133.

[34] J.P. Perdew, K. Burke, M. Ernzerhof, Generalized gradient approximation made simple, Phys. Rev. Lett. 77 (1996) 3865.

[35] M. Dion, H. Rydberg, E. Schröder, D.C. Langreth, B.I. Lundqvist, Van der waals density functional for general geometries, Phys. Rev. Lett. 92 (2004), 246401.

[36] G. Román-Pérez, J.M. Soler, Efficient implementation of a van der waals density functional: application to double-wall carbon nanotubes, Phys. Rev. Lett. 103 (2009), 096102.

[37] J.M. Soler, E. Artacho, J.D. Gale, A. García, J. Junquera, P. Ordejón, D. SánchezPortal, The siesta method for ab initio order-n materials simulation, J. Phys. Condens. Matter 14 (2002) 2745.

[38] N. Troullier, J.L. Martins, Efficient pseudopotentials for plane-wave calculations, Phys. Rev. B 43 (1991) 1993.

[39] H.J. Monkhorst, J.D. Pack, Special points for brillouin-zone integrations, Phys Rev. B 13 (1976) 5188-5192.

[40] S. Datta, Electronic Transport in Mesoscopic Systems, Cambridge Studies in Semiconductor Physics, Cambridge University Press, 1997.

[41] Y. Xue, S. Datta, M.A. Ratner, First-principles based matrix green's function approach to molecular electronic devices: general formalism, Chem. Phys. 281 (2002) 151-170.

[42] C. Caroli, R. Combescot, P. Nozieres, D. Saint-James, Direct calculation of the tunneling current, J. Phys. C Solid State Phys. 4 (1971) 916-929.

[43] R. Landauer, Spatial variation of currents and fields due to localized scatterers in metallic conduction, IBM J. Res. Dev. 1 (1957) 223-231.

[44] R.S. Fisher, P.A. Lee, Relation between conductivity and transmission matrix, Phys. Rev. B 23 (1981) 6851.

[45] N. Okabayashi, M. Paulsson, H. Ueba, Y. Konda, T. Komeda, Inelastic tunneling spectroscopy of alkanethiol molecules: high-resolution spectroscopy and theoretical simulations, Phys. Rev. Lett. 104 (2010), 077801.

[46] M. Paulsson, M. Brandbyge, Transmission eigenchannels from nonequilibrium greens functions, Phys. Rev. B 76 (2007), 115117.

[47] Q. He, S. Wu, Z. Yin, H. Zhang, Graphene-based electronic sensors, Chem. Sci. 3 (2012) 1764.

[48] H. Karamitaheri, N. Neophytou, M. Pourfath, R. Faez, H. Kosina, Engineering enhanced thermoelectric properties in zigzag graphene nanoribbons, J. Appl. Phys. 111 (2012) 054501-054510.

[49] N. Ansari, F. Nazari, F. Illas, Line defects and induced doping effects in graphene, hexagonal boron nitride and hybrid bnc, Phys. Chem. Chem. Phys. 16 (2014) 21473-21485.

[50] D. Gunlycke, C.T. White, Graphene valley filter using a line defect, Phys. Rev. Lett. 106 (2011), 136806.

[51] R.F.W. Bader, Atoms in Molecules: a Quantum Theory, Oxford University Press, New York, 1990.

[52] W. Tang, E. Sanville, G. Henkelman, A grid-based Bader analysis algorithm without lattice bias, J. Phys. Condens. Matter 21 (2009) 084204.

[53] F. Mehmood, R. Pachter, W. Lu, J.J. Boeckl, Adsorption and diffusion of oxygen on single-layer graphene with topological defects, J. Phys. Colloid Chem. 117 (2013) 10366-10374.

[54] J. Prasongkit, G.T. Feliciano, A.R. Rocha, Y. He, T. Osotchan, R. Ahuja R.H. Scheicher, Theoretical assessment of feasibility to sequence DNA through interlayer electronic tunneling transport at aligned nanopores in bilayer graphene, Sci. Rep. 5 (2015), 17560. 